\title{
Chemically Accurate Excitation Energies With Small Basis Sets
}

\author{
Emmanuel Giner, ${ }^{1, a)}$ Anthony Scemama, ${ }^{2}$ Julien Toulouse, ${ }^{1}$ and Pierre-François Loos ${ }^{2}$, b) \\ 1) Laboratoire de Chimie Théorique (UMR 7616), Sorbonne Université, CNRS, Paris, France \\ 2) Laboratoire de Chimie et Physique Quantiques (UMR 5626), Université de Toulouse, CNRS, UPS, France
}


double- $\zeta$ basis sets. We illustrate the present approach on various types of excited states (valence, Rydberg, and double excitations) in several small organic molecules (methylene, water, ammonia, carbon dimer and ethylene). The present study clearly evidences that special care has to be taken with very diffuse excited states where the present correction does not catch the radial incompleteness of the one-electron basis set.

\section{INTRODUCTION}

One of the most fundamental problems of conventional wave function electronic structure methods is their slow energy convergence with respect to the size of the one-electron basis set. The overall basis-set incompleteness error can be, qualitatively at least, split in two contributions stemming from the radial and angular incompleteness. Although for ground-state properties angular incompleteness is by far the main source of error, it is definitely not unusual to have a significant radial incompleteness in the case of excited states (especially for Rydberg states), which can be alleviated by using additional sets of diffuse basis functions (i.e. augmented basis sets).

Explicitly-correlated F12 methods ${ }^{1-3}$ have been specifically designed to efficiently catch angular incompleteness. ${ }^{4-9} \mathrm{Al}-$ though they have been extremely successful to speed up convergence of ground-state energies and properties, such as correlation and atomization energies, ${ }^{10}$ their performance for excited states ${ }^{11-18}$ has been much more conflicting. ${ }^{11,12}$

Instead of F12 methods, here we propose to follow a different route and investigate the performance of the recently proposed density-based basis set incompleteness correction. ${ }^{19}$ Contrary to our recent study on atomization and correlation energies, ${ }^{20}$ the present contribution focuses on vertical and adiabatic excitation energies in molecular systems which is a much tougher test for the reasons mentioned above. This density-based correction relies on short-range correlation density functionals (with multideterminant reference) from

\footnotetext{
a)Corresponding author: emmanuel.giner@lct.jussieu.fr

b) Corresponding author: loos@irsamc.ups-tlse.fr
}

range-separated density-functional theory ${ }^{21-36}$ (RS-DFT) to capture the missing part of the short-range correlation effects, a consequence of the incompleteness of the one-electron basis set. Because RS-DFT combines rigorously density-functional theory (DFT) ${ }^{37}$ and wave function theory (WFT) ${ }^{38}$ via a decomposition of the electron-electron interaction into a nondivergent long-range part and a (complementary) short-range part (treated with WFT and DFT, respectively), the WFT method is relieved from describing the short-range part of the correlation hole around the electron-electron coalescence points (the so-called electron-electron cusp). ${ }^{39}$ Consequently, the energy convergence with respect to the size of the basis set is significantly improved, ${ }^{40}$ and chemical accuracy can be obtained even with small basis sets. For example, in Ref. 20, we have shown that one can recover quintuple- $\zeta$ quality atomization and correlation energies with triple- $\zeta$ basis sets for a much lower computational cost than F12 methods.

This work is organized as follows. In Sec. II, the main working equations of the density-based correction are reported and discussed. Computational details are given in Sec. III. In Sec. IV, we discuss our results for each system and draw our conclusions in Sec. V. Unless otherwise stated, atomic units are used.

\section{THEORY}

The present basis-set correction assumes that we have, in a given (finite) basis set $\mathcal{B}$, the ground-state and the $k$ th excitedstate energies, $E_{0}^{\mathcal{B}}$ and $E_{k}^{\mathcal{B}}$, their one-electron densities, $n_{k}^{\mathcal{B}}(\mathbf{r})$ and $n_{0}^{\mathcal{B}}(\mathbf{r})$, as well as their opposite-spin on-top pair densities, $n_{2,0}^{\mathcal{B}}(\mathbf{r})$ and $n_{2, k}^{\mathcal{B}}(\mathbf{r})$, Therefore, the complete-basis-set (CBS) energy of the ground and excited states may be approximated 
$\operatorname{as}^{19}$

$$
\begin{aligned}
& E_{0}^{\mathrm{CBS}} \approx E_{0}^{\mathcal{B}}+\bar{E}^{\mathcal{B}}\left[n_{0}^{\mathcal{B}}\right], \\
& E_{k}^{\mathrm{CBS}} \approx E_{k}^{\mathcal{B}}+\bar{E}^{\mathcal{B}}\left[n_{k}^{\mathcal{B}}\right],
\end{aligned}
$$

where

$$
\bar{E}^{\mathcal{B}}[n]=\min _{\Psi \rightsquigarrow n}\left\langle\Psi\left|\hat{T}+\hat{W}_{\mathrm{ee}}\right| \Psi\right\rangle-\min _{\Psi^{\mathcal{B}} \rightsquigarrow n}\left\langle\Psi^{\mathcal{B}}\left|\hat{T}+\hat{W}_{\mathrm{ee}}\right| \Psi^{\mathcal{B}}\right\rangle
$$

is the basis-dependent complementary density functional,

$$
\hat{T}=-\frac{1}{2} \sum_{i}^{N} \nabla_{i}^{2}, \quad \hat{W}_{\mathrm{ee}}=\sum_{i<j}^{N} r_{i j}^{-1},
$$

are the kinetic and electron-electron repulsion operators, respectively, and $\Psi^{\mathcal{B}}$ and $\Psi$ are two general $N$-electron normalized wave functions belonging to the Hilbert spaces spanned by $\mathcal{B}$ and the complete basis, respectively. The notation $\Psi \rightsquigarrow n$ in Eq. (2) states that $\Psi$ yields the one-electron density $n$.

Hence, the CBS excitation energy associated with the $k$ th excited state reads

$$
\begin{aligned}
\Delta E_{k}^{\mathrm{CBS}} & =E_{k}^{\mathrm{CBS}}-E_{0}^{\mathrm{CBS}} \\
& \approx \Delta E_{k}^{\mathcal{B}}+\Delta \bar{E}^{\mathcal{B}}\left[n_{0}^{\mathcal{B}}, n_{k}^{\mathcal{B}}\right],
\end{aligned}
$$

where

$$
\Delta E_{k}^{\mathcal{B}}=E_{k}^{\mathcal{B}}-E_{0}^{\mathcal{B}}
$$

is the excitation energy in $\mathcal{B}$ and

$$
\Delta \bar{E}^{\mathcal{B}}\left[n_{0}^{\mathcal{B}}, n_{k}^{\mathcal{B}}\right]=\bar{E}^{\mathcal{B}}\left[n_{k}^{\mathcal{B}}\right]-\bar{E}^{\mathcal{B}}\left[n_{0}^{\mathcal{B}}\right]
$$

its basis-set correction. An important property of the present correction is

$$
\lim _{\mathcal{B} \rightarrow \mathrm{CBS}} \Delta \bar{E}^{\mathcal{B}}\left[n_{0}^{\mathcal{B}}, n_{k}^{\mathcal{B}}\right]=0 .
$$

In other words, the correction vanishes in the CBS limit, hence guaranteeing an unaltered limit. ${ }^{20}$ Note that in Eqs. (1a) and (1b) we have assumed that the same density functional $\bar{E}^{\mathcal{B}}$ can be used for correcting all excited-state energies, which seems a reasonable approximation since the electron-electron cusp effects are largely universal. ${ }^{1,41-45}$

\section{A. Range-separation function}

As initially proposed in Ref. 19 and further developed in Ref. 20, we have shown that one can efficiently approximate $\bar{E}^{\mathcal{B}}[n]$ by short-range correlation functionals with multideterminantal (ECMD) reference borrowed from RS-DFT. ${ }^{46}$ The ECMD functional, $\bar{E}_{\mathrm{c}, \mathrm{md}}^{\mathrm{sr}}[n, \mu]$, is a function of the rangeseparation parameter $\mu$ and admits, for any $n$, the following two limits

$$
\begin{aligned}
\lim _{\mu \rightarrow \infty} \bar{E}_{\mathrm{c}, \mathrm{md}}^{\mathrm{sr}}[n, \mu] & =0, \\
\lim _{\mu \rightarrow 0} \bar{E}_{\mathrm{c}, \mathrm{md}}^{\mathrm{sr}}[n, \mu] & =E_{\mathrm{c}}[n],
\end{aligned}
$$

which correspond to the WFT limit $(\mu \rightarrow \infty)$ and the KohnSham DFT (KS-DFT) limit $(\mu=0)$. In Eq. (8b), $E_{\mathrm{c}}[n]$ is the usual universal correlation density functional defined in KSDFT. ${ }^{47,48}$

The key ingredient that allows us to exploit ECMD functionals for correcting the basis-set incompleteness error is the range-separated function

$$
\mu^{\mathcal{B}}(\mathbf{r})=\frac{\sqrt{\pi}}{2} W^{\mathcal{B}}(\mathbf{r}, \mathbf{r}),
$$

which automatically adapts to the spatial non-homogeneity of the basis-set incompleteness error. It is defined such that the long-range interaction of RS-DFT, $w^{\operatorname{lr}, \mu}\left(r_{12}\right)=\operatorname{erf}\left(\mu r_{12}\right) / r_{12}$, coincides, at coalescence, with an effective two-electron interaction $W^{\mathcal{B}}\left(\mathbf{r}_{1}, \mathbf{r}_{2}\right)$ "mimicking" the Coulomb operator in an incomplete basis $\mathcal{B}$, i.e. $w^{\mathrm{lr}, \mu^{\mathcal{B}}(\mathbf{r})}(0)=W^{\mathcal{B}}(\mathbf{r}, \mathbf{r})$ at any $\mathbf{r}^{19}$ The explicit expression of $W^{\mathcal{B}}\left(\mathbf{r}_{1}, \mathbf{r}_{2}\right)$ is given by

$$
W^{\mathcal{B}}\left(\mathbf{r}_{1}, \mathbf{r}_{2}\right)= \begin{cases}f^{\mathcal{B}}\left(\mathbf{r}_{1}, \mathbf{r}_{2}\right) / n_{2}^{\mathcal{B}}\left(\mathbf{r}_{1}, \mathbf{r}_{2}\right), & \text { if } n_{2}^{\mathcal{B}}\left(\mathbf{r}_{1}, \mathbf{r}_{2}\right) \neq 0 \\ \infty, & \text { otherwise }\end{cases}
$$

where

$$
n_{2}^{\mathcal{B}}\left(\mathbf{r}_{1}, \mathbf{r}_{2}\right)=\sum_{p q r s \in \mathcal{B}} \phi_{p}\left(\mathbf{r}_{1}\right) \phi_{q}\left(\mathbf{r}_{2}\right) \Gamma_{p q}^{r s} \phi_{r}\left(\mathbf{r}_{1}\right) \phi_{s}\left(\mathbf{r}_{2}\right),
$$

and $\Gamma_{p q}^{r s}=2\left\langle\Psi^{\mathcal{B}}\left|\hat{a}_{r_{\downarrow}}^{+} \hat{a}_{s_{\uparrow}}^{+} \hat{a}_{q_{\uparrow}} \hat{a}_{p_{\downarrow}}\right| \Psi^{\mathcal{B}}\right\rangle$ are the opposite-spin pair density associated with $\Psi^{\mathcal{B}}$ and its corresponding tensor, respectively, $\phi_{p}(\mathbf{r})$ is a (real-valued) molecular orbital (MO),

$$
f^{\mathcal{B}}\left(\mathbf{r}_{1}, \mathbf{r}_{2}\right)=\sum_{p q r s t u \in \mathcal{B}} \phi_{p}\left(\mathbf{r}_{1}\right) \phi_{q}\left(\mathbf{r}_{2}\right) V_{p q}^{r s} \Gamma_{r s}^{t u} \phi_{t}\left(\mathbf{r}_{1}\right) \phi_{u}\left(\mathbf{r}_{2}\right),
$$

and $V_{p q}^{r s}=\langle p q \mid r s\rangle$ are two-electron Coulomb integrals. An important feature of $W^{\mathcal{B}}\left(\mathbf{r}_{1}, \mathbf{r}_{2}\right)$ is that it tends to the regular Coulomb operator $r_{12}^{-1}$ as $\mathcal{B} \rightarrow \mathrm{CBS}$, which implies that

$$
\lim _{\mathcal{B} \rightarrow \mathrm{CBS}} \mu^{\mathcal{B}}(\mathbf{r})=\infty,
$$

ensuring that $\bar{E}^{\mathcal{B}}[n]$ vanishes when $\mathcal{B}$ is complete. We refer the interested readers to Refs. 19 and 20 for additional details.

\section{B. Short-range correlation functionals}

The local-density approximation (LDA) of the ECMD complementary functional is defined as

$$
\bar{E}_{\mathrm{LDA}}^{\mathcal{B}}\left[n, \mu^{\mathcal{B}}\right]=\int n(\mathbf{r}) \bar{\varepsilon}_{\mathrm{c}, \mathrm{md}}^{\mathrm{s}, \mathrm{LDA}}\left(n(\mathbf{r}), \zeta(\mathbf{r}), \mu^{\mathcal{B}}(\mathbf{r})\right) d \mathbf{r},
$$

where $\zeta=\left(n_{\uparrow}-n_{\downarrow}\right) / n$ is the spin polarization and $\bar{\varepsilon}_{\mathrm{c}, \mathrm{md}}^{\mathrm{sr} \mathrm{LDA}}(n, \zeta, \mu)$ is the ECMD short-range correlation energy per electron of the uniform electron gas (UEG) ${ }^{49}$ parameterized in Ref. 27. 
The functional $\bar{\varepsilon}_{\mathrm{c}, \mathrm{md}}^{\mathrm{sr}, \mathrm{LDA}}$ from Eq. (14) presents two main defects: i) at small $\mu$, it overestimates the correlation energy, and ii) UEG-based quantities are hardly transferable when the system becomes strongly correlated. An attempt to solve these problems was suggested by some of the authors in the context of RS-DFT. ${ }^{36}$ They proposed to interpolate between the usual Perdew-Burke-Ernzerhof (PBE) correlation functional ${ }^{50}$ $\varepsilon_{\mathrm{c}}^{\mathrm{PBE}}(n, s, \zeta)$ (where $s=\nabla n / n^{4 / 3}$ is the reduced density gradient) at $\mu=0$ and the exact large- $\mu$ behavior. ${ }^{23,27,51}$ In the context of RS-DFT, the large- $\mu$ behavior corresponds to an extremely short-range interaction in the short-range functional. In this regime, the ECMD energy

$$
\bar{E}_{\mathrm{c}, \mathrm{md}}^{\mathrm{sr}}=\frac{2 \sqrt{\pi}(1-\sqrt{2})}{3 \mu^{3}} \int d \mathbf{r} n_{2}(\mathbf{r})+\mathcal{O}\left(\mu^{-4}\right)
$$

only depends on the exact on-top pair density $n_{2}(\mathbf{r}) \equiv$ $n_{2}(\mathbf{r}, \mathbf{r})$ which is obtained from the exact ground-state wave function $\Psi$ belonging to the many-electron Hilbert space in the CBS limit.

Obviously, an exact quantity such as $n_{2}(\mathbf{r})$ is out of reach in practical calculations and must be approximated by a function referred here as $\tilde{n}_{2}(\mathbf{r})$. For a given $\tilde{n}_{2}(\mathbf{r})$, some of the authors proposed the following functional form in order to interpolate between $\varepsilon_{\mathrm{c}}^{\mathrm{PBE}}(n, s, \zeta)$ at $\mu=0$ and Eq. (15) as $\mu \rightarrow \infty:^{36}$

$$
\begin{aligned}
& \bar{\varepsilon}_{\mathrm{c}, \mathrm{md}}^{\mathrm{s}, \mathrm{PBE}}\left(n, \tilde{n}_{2}, s, \zeta, \mu\right)=\frac{\varepsilon_{\mathrm{c}}^{\mathrm{PBE}}(n, s, \zeta)}{1+\beta^{\mathrm{PBE}}\left(n, \tilde{n}_{2}, s, \zeta\right) \mu^{3}}, \\
& \beta^{\mathrm{PBE}}\left(n, \tilde{n}_{2}, s, \zeta\right)=\frac{3}{2 \sqrt{\pi}(1-\sqrt{2})} \frac{\varepsilon_{\mathrm{c}}^{\mathrm{PBE}}(n, s, \zeta)}{\tilde{n}_{2} / n} .
\end{aligned}
$$

As illustrated in the context of RS-DFT, ${ }^{36}$ such a functional form is able to treat both weakly and strongly correlated systems thanks to the explicit inclusion of $\varepsilon_{\mathrm{c}}^{\mathrm{PBE}}$ and $\tilde{n}_{2}$, respectively. Therefore, in the present context, we introduce the general form of the PBE-based complementary functional within a given basis set $\mathcal{B}$

$$
\begin{aligned}
\bar{E}_{\mathrm{PBE}}^{\mathcal{B}}\left[n, \tilde{n}_{2}, \mu^{\mathcal{B}}\right]=\int n(\mathbf{r}) \\
\quad \times \bar{\varepsilon}_{\mathrm{c}, \mathrm{sr}, \mathrm{PBE}}\left(n(\mathbf{r}), \tilde{n}_{2}(\mathbf{r}), s(\mathbf{r}), \zeta(\mathbf{r}), \mu^{\mathcal{B}}(\mathbf{r})\right) d \mathbf{r},
\end{aligned}
$$

which has an explicit dependency on both the rangeseparation function $\mu^{\mathcal{B}}(\mathbf{r})$ (instead of the range-separation parameter in RS-DFT) and the approximation level of $\tilde{n}_{2}$.

In Ref. 20, some of the authors introduced a version of the PBE-based functional, here-referred as PBE-UEG

$$
\bar{E}_{\mathrm{PBE}-\mathrm{UEG}}^{\mathcal{B}} \equiv \bar{E}_{\mathrm{PBE}}^{\mathcal{B}}\left[n, n_{2}^{\mathrm{UEG}}, \mu^{\mathcal{B}}\right]
$$

in which the on-top pair density was approximated by its UEG version, i.e., $\tilde{n}_{2}(\mathbf{r})=n_{2}^{\mathrm{UEG}}(\mathbf{r})$, with

$$
n_{2}^{\mathrm{UEG}}(\mathbf{r}) \approx n(\mathbf{r})^{2}\left[1-\zeta(\mathbf{r})^{2}\right] g_{0}(n(\mathbf{r})),
$$

and where $g_{0}(n)$ is the UEG on-top pair distribution function [see Eq. (46) of Ref. 51]. Note that in Eq. (19) the dependence on the spin polarization $\zeta$ is only approximate. As illustrated in Ref. 20, the PBE-UEG functional has clearly shown, for weakly correlated systems, to improve energetics over the pure UEG-based functional $\bar{E}_{\mathrm{LDA}}^{\mathcal{B}}$ [see Eq. (14)] thanks to the leverage brought by the PBE functional in the small- $\mu$ regime.

However, the underlying UEG on-top pair density might not be suited for the treatment of excited states and/or strongly correlated systems. Besides, in the context of the present basis-set correction, $n_{2}^{\mathcal{B}}(\mathbf{r})$, the on-top pair density in $\mathcal{B}$, must be computed anyway to obtain $\mu^{\mathcal{B}}$ (r) [see Eqs. (9) and (10)]. Therefore, as in Ref. 36, we define a better approximation of the exact on-top pair density as

$$
\stackrel{\circ}{2}_{2}^{\mathcal{B}}(\mathbf{r})=n_{2}^{\mathcal{B}}(\mathbf{r})\left(1+\frac{2}{\sqrt{\pi} \mu^{\mathcal{B}}(\mathbf{r})}\right)^{-1}
$$

which directly follows from the large- $\mu$ extrapolation of the exact on-top pair density proposed by Gori-Giorgi and Savin ${ }^{51}$ in the context of RS-DFT. Using this new ingredient, we propose here the "PBE-ontop" (PBEot) functional

$$
\bar{E}_{\mathrm{PBEot}}^{\mathcal{B}} \equiv \bar{E}_{\mathrm{PBE}}^{\mathcal{B}}\left[n, \stackrel{n}{2}_{2}^{\mathcal{B}}, \mu^{\mathcal{B}}\right] \text {. }
$$

The sole distinction between PBE-UEG and PBEot is the level of approximation of the exact on-top pair density.

\section{COMPUTATIONAL DETAILS}

In the present study, we compute the ground- and excitedstate energies, one-electron densities and on-top pair densities with a selected configuration interaction (sCI) method known as CIPSI (Configuration Interaction using a Perturbative Selection made Iteratively). ${ }^{52-54}$ Both the implementation of the CIPSI algorithm and the computational protocol for excited states is reported in Ref. 55. The total energy of each state is obtained via an efficient extrapolation procedure of the $\mathrm{sCI}$ energies designed to reach near-FCI accuracy. ${ }^{56,57}$ These energies will be labeled exFCI in the following. Using near-FCI excitation energies (within a given basis set) has the indisputable advantage to remove the error inherent to the WFT method. Indeed, in the present case, the only source of error on the excitation energies is due to basis-set incompleteness. We refer the interested reader to Refs. 56-61 for more details. The one-electron densities and on-top pair densities are computed from a very large CIPSI expansion containing up to several million of Slater determinants. All the RS-DFT and exFCI calculations have been performed with QUANTUM PACKAGE. ${ }^{57}$ For the numerical quadratures, we employ the SG-2 grid. ${ }^{62}$ Except for methylene for which FCI/TZVP geometries have been taken from Ref. 63, the other molecular geometries have been extracted from Refs. 59 and 61 and have been obtained at the CC3/aug-cc-pVTZ level of theory. For the sake of completeness, all these geometries are reported in the supporting information. Frozen-core calculations are systematically performed and defined as such: a He core is frozen from $\mathrm{Li}$ to $\mathrm{Ne}$, while a $\mathrm{Ne}$ core is frozen from $\mathrm{Na}$ to Ar. The frozen-core density-based correction is used consistently with the frozen-core approximation in WFT methods. We refer 
the reader to Ref. 20 for an explicit derivation of the equations associated with the frozen-core version of the present density-based basis-set correction. Compared to the exFCI calculations performed to compute energies and densities, the basis-set correction represents, in any case, a marginal computational cost. In the following, we employ the AVXZ shorthand notations for Dunning's aug-cc-pVXZ basis sets.

\section{RESULTS AND DISCUSSION}

\section{A. Methylene}

Methylene is a paradigmatic system in electronic structure theory. ${ }^{64}$ Due to its relative small size, its ground and excited states have been thoroughly studied with high-level ab initio methods. ${ }^{63-72}$

As a first test of the present density-based basis-set correction, we consider the four lowest-lying states of methylene $\left(1^{3} B_{1}, 1^{1} A_{1}, 1^{1} B_{1}\right.$ and $\left.2^{1} A_{1}\right)$ at their respective equilibrium geometry and compute the corresponding adiabatic transition energies for basis sets ranging from AVDZ to AVQZ. We have also computed total energies at the exFCI/AV5Z level and used these alongside the quadruple- $\zeta$ ones to extrapolate the total energies to the CBS limit with the usual extrapolation formula $^{73}$

$$
E^{\mathrm{AVXZ}}(\mathrm{X})=E^{\mathrm{CBS}}+\alpha \mathrm{X}^{-3} .
$$

These results are illustrated in Fig. 1 and reported in Table I alongside reference values from the literature obtained with various deterministic and stochastic approaches. ${ }^{63,66,70,72} \mathrm{To}-$ tal energies for each state can be found in the supporting information. The exFCI/CBS values are still off by a few tenths of a $\mathrm{kcal} / \mathrm{mol}$ compared to the DMC results of Zimmerman et $\mathrm{al}^{70}$ which are extremely close from the experimentallyderived adiabatic energies. The reason of this discrepancy is probably due to the frozen-core approximation which has been applied in our case and has shown to significantly affect adiabatic energies. ${ }^{74,75}$ However, the exFCI/CBS energies are in perfect agreement with the semistochastic heat-bath CI (SHCI) calculations from Ref. 72, as expected.

Figure 1 clearly shows that, for the double- $\zeta$ basis, the exFCI adiabatic energies are far from being chemically accurate with errors as high as $0.15 \mathrm{eV}$. From the triple- $\zeta$ basis onward, the exFCI excitation energies are chemically accurate though (i.e. error below $1 \mathrm{kcal} / \mathrm{mol}$ or $0.043 \mathrm{eV}$ ), and converge steadily to the CBS limit when one increases the size of the basis set. Concerning the basis-set correction, already at the double- $\zeta$ level, the PBEot correction returns chemically accurate excitation energies. The performance of the PBE-UEG and LDA functionals is less impressive. Yet, they still yield significant reductions of the basis-set incompleteness error, hence representing a good compromise between computational cost and accuracy. Note that the results for the PBE-UEG functional are not represented in Fig. 1 as they are very similar to the LDA ones (similar considerations apply to the other systems studied below). It is also quite evident that, the basis-set correction
TABLE I. Adiabatic transition energies (in eV) of excited states of methylene for various methods and basis sets. The relative difference with respect to the exFCI/CBS result is reported in square brackets. See supporting information for total energies.

\begin{tabular}{|c|c|c|c|c|}
\hline \multirow[b]{2}{*}{ Method } & \multirow[b]{2}{*}{ Basis set } & \multicolumn{3}{|c|}{ Transitions } \\
\hline & & $1^{3} B_{1} \rightarrow 1^{1} A_{1}$ & $1^{3} B_{1} \rightarrow 1^{1} B_{1}$ & $1^{3} B_{1} \rightarrow 2^{1} A_{1}$ \\
\hline \multirow[t]{5}{*}{ exFCI } & AVDZ & $0.441[+0.057]$ & $1.536[+0.152]$ & $2.659[+0.162]$ \\
\hline & AVTZ & $0.408[+0.024]$ & $1.423[+0.040]$ & $2.546[+0.049]$ \\
\hline & AVQZ & $0.395[+0.011]$ & $1.399[+0.016]$ & $2.516[+0.020]$ \\
\hline & AV5Z & $0.390[+0.006]$ & $1.392[+0.008]$ & $2.507[+0.010]$ \\
\hline & CBS & 0.384 & 1.384 & 2.497 \\
\hline \multirow[t]{3}{*}{ exFCI+PBEot } & AVDZ & $0.347[-0.037]$ & $1.401[+0.017]$ & $2.511[+0.014]$ \\
\hline & AVTZ & $0.374[-0.010]$ & $1.378[-0.006]$ & $2.491[-0.006]$ \\
\hline & AVQZ & $0.379[-0.005]$ & $1.378[-0.006]$ & $2.489[-0.008]$ \\
\hline \multirow[t]{3}{*}{ exFCI+PBE-UEG } & AVDZ & $0.308[-0.076]$ & $1.388[+0.004]$ & $2.560[+0.064]$ \\
\hline & AVTZ & $0.356[-0.028]$ & $1.371[-0.013]$ & $2.510[+0.013]$ \\
\hline & AVQZ & $0.371[-0.013]$ & $1.375[-0.009]$ & $2.498[+0.002]$ \\
\hline \multirow[t]{3}{*}{ exFCI+LDA } & AVDZ & $0.337[-0.047]$ & $1.420[+0.036]$ & $2.586[+0.089]$ \\
\hline & AVTZ & $0.359[-0.025]$ & $1.374[-0.010]$ & $2.514[+0.017]$ \\
\hline & AVQZ & $0.370[-0.014]$ & $1.375[-0.009]$ & $2.499[-0.002]$ \\
\hline $\mathrm{SHCI}^{\mathrm{a}}$ & AVQZ & 0.393 & 1.398 & 2.516 \\
\hline CR-EOMCC $(2,3) \mathrm{D}^{\mathrm{b}}$ & AV5Z & 0.430 & 1.464 & 2.633 \\
\hline $\mathrm{FCI}^{\mathrm{c}}$ & $\mathrm{TZ2P}$ & 0.483 & 1.542 & 2.674 \\
\hline $\mathrm{DMC}^{\mathrm{d}}$ & & 0.406 & 1.416 & 2.524 \\
\hline Exp. $^{e}$ & & 0.406 & 1.415 & \\
\hline
\end{tabular}

a Semistochastic heat-bath CI (SHCI) calculations from Ref. 72.

${ }^{\mathrm{b}}$ Completely-renormalized equation-of-motion coupled cluster (CR-EOMCC) calculations from Refs. 71.

${ }^{c}$ Reference 63.

${ }^{\mathrm{d}}$ Diffusion Monte Carlo (DMC) calculations from Ref. 70 obtained with a CAS $(6,6)$ trial wave function.

${ }^{\mathrm{e}}$ Experimentally-derived values. See footnotes of Table II from Ref. 71 for additional details.

has the tendency of over-correcting the excitation energies via an over-stabilization of the excited states compared to the ground state. This trend is quite systematic as we shall see below.

\section{B. Rydberg States of Water and Ammonia}

For the second test, we consider the water ${ }^{55,59,60,76-78}$ and ammonia ${ }^{59,79,80}$ molecules. They are both well studied and possess Rydberg excited states which are highly sensitive to the radial completeness of the one-electron basis set, as evidenced in Ref. 59. Table II reports vertical excitation energies for various singlet and triplet excited states of water and ammonia at various levels of theory (see the supporting information for total energies). The basis-set corrected theoretical best estimates (TBEs) have been extracted from Ref. 59 and have been obtained on the same geometries. These results are also depicted in Figs. 2 and 3 for $\mathrm{H}_{2} \mathrm{O}$ and $\mathrm{NH}_{3}$, respectively. One would have noticed that the basis-set effects are particularly strong for the third singlet excited state of water and the third and fourth singlet excited states of ammonia where this effect is even magnified. In other words, substantial error remains in these cases even with the largest AVQZ basis set. In these cases, one really needs doubly augmented basis sets to reach radial completeness. The first observation worth reporting is that all three RS-DFT correlation functionals have very similar 

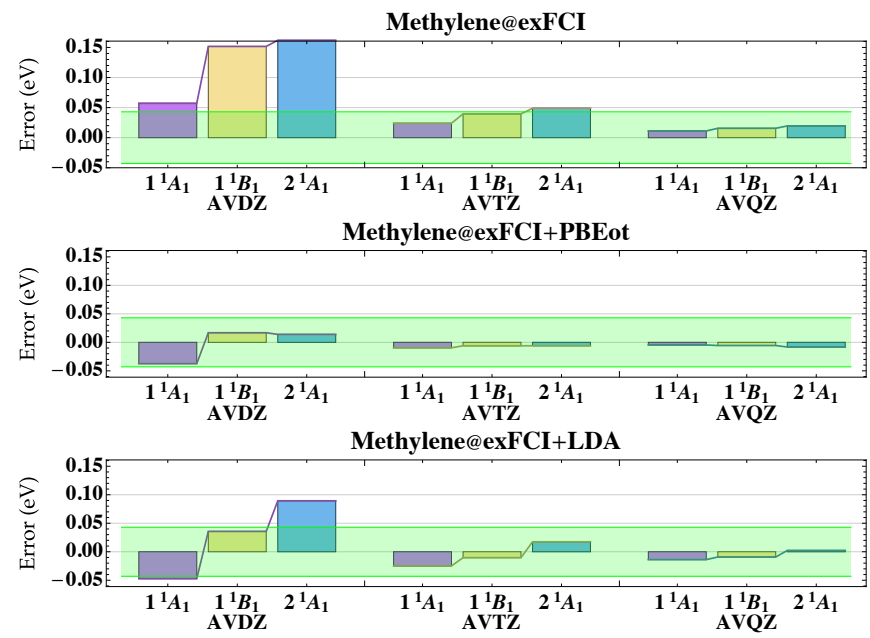

FIG. 1. Error in adiabatic excitation energies (in eV) of methylene for various basis sets and methods. The green region corresponds to chemical accuracy (i.e., error below $1 \mathrm{kcal} / \mathrm{mol}$ or $0.043 \mathrm{eV}$ ). See Table I for raw data.
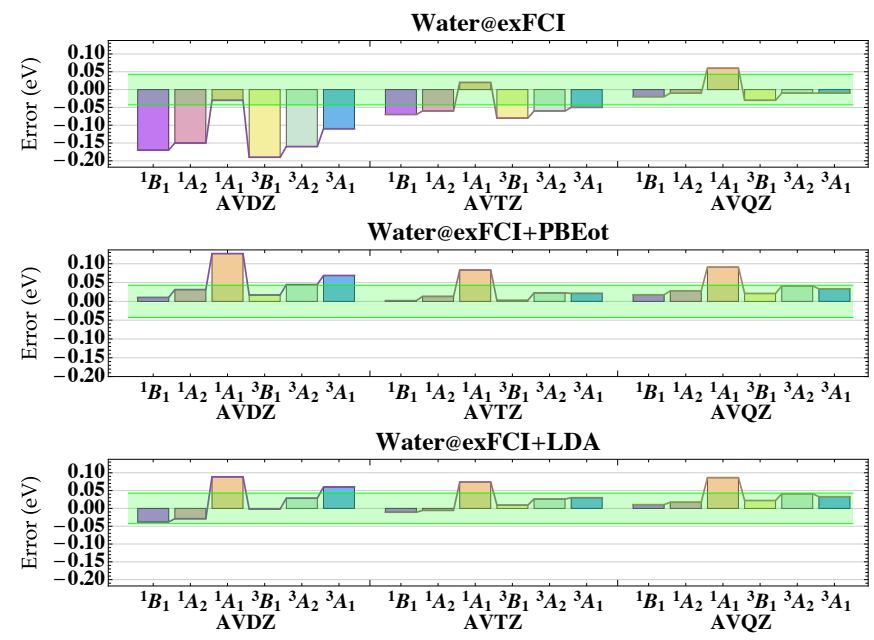

FIG. 2. Error in vertical excitation energies (in $\mathrm{eV}$ ) of water for various basis sets and methods. The green region corresponds to chemical accuracy (i.e., error below $1 \mathrm{kcal} / \mathrm{mol}$ or $0.043 \mathrm{eV}$ ). See the supporting information for raw data.

behaviors and they significantly reduce the error on the excitation energies for most of the states. However, these results also clearly evidence that special care has to be taken for very diffuse excited states where the present correction cannot catch the radial incompleteness of the one-electron basis set, a feature which is far from being a cusp-related effect.

\section{Doubly-Excited States of the Carbon Dimer}

In order to have a miscellaneous test set of excitations, in a third time, we propose to study some doubly-excited states of the carbon dimer $\mathrm{C}_{2}$, a prototype system for strongly correlated and multireference systems. ${ }^{56,68,69,81-87}$ These two
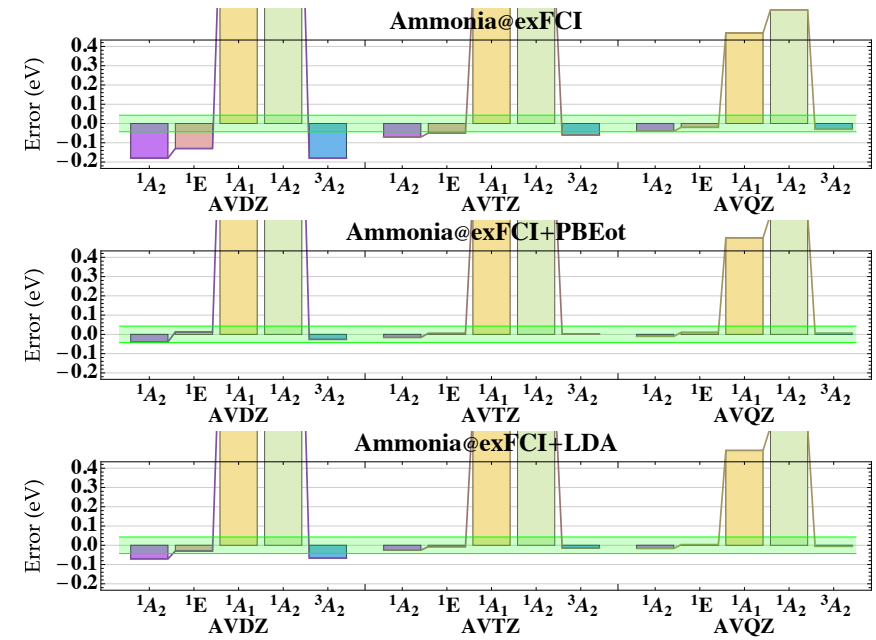

FIG. 3. Error in vertical excitation energies (in $\mathrm{eV}$ ) of ammonia for various basis sets and methods. The green region corresponds to chemical accuracy (i.e., error below $1 \mathrm{kcal} / \mathrm{mol}$ or $0.043 \mathrm{eV}$ ). See the supporting information for raw data.

valence excitations $-1{ }^{1} \Sigma_{g}^{+} \rightarrow 1^{1} \Delta_{g}$ and $1^{1} \Sigma_{g}^{+} \rightarrow 2^{1} \Sigma_{g}^{+}$ - are both of $(\pi, \pi) \rightarrow(\sigma, \sigma)$ character. They have been recently studied with state-of-the-art methods, and have been shown to be "pure" doubly-excited states as they involve an insignificant amount of single excitations. ${ }^{61}$ The vertical excitation energies associated with these transitions are reported in Table II and represented in Fig. 4. An interesting point here is that one really needs to consider the PBEot functional to get chemically accurate excitation energies with the AVDZ atomic basis set. We believe that the present result is a direct consequence of the multireference character of the $\mathrm{C}_{2}$ molecule. In other words, the UEG on-top pair density used in the LDA and PBE-UEG functionals (see Sec. II B) is a particularly bad approximation of the true on-top pair density for the present system.

It is interesting to study the behavior of the key quantities involved in the basis-set correction for different states as the basis-set incompleteness error is obviously state specific. In Fig. 5, we report $\mu^{\mathcal{B}}(z), n^{\mathcal{B}}(z) \bar{\varepsilon}_{\mathrm{c}, \mathrm{md}}^{\mathrm{sr}, \mathrm{PBEot}}(z)$, and $n_{2}^{\mathcal{B}}(z)$ along the nuclear axis $(z)$ for the two ${ }^{1} \Sigma_{g}^{+}$electronic states of $C_{2}$ computed with the AVDZ, AVTZ, and AVQZ basis sets. The graphs gathered in Fig. 5 illustrate several general features regarding the present basis-set correction:

- the maximal values of $\mu^{\mathcal{B}}(z)$ are systematically close to the nuclei, a signature of the atom-centered basis set;

- the overall magnitude of $\mu^{\mathcal{B}}(z)$ increases with the basis set, which reflects the improvement of the description of the correlation effects when enlarging the basis set;

- the absolute value of the energetic correction decreases when the size of the basis set increases;

- there is a clear correspondence between the values of the energetic correction and the on-top pair density. 
TABLE II. Vertical excitation energies (in eV) of excited states of water, ammonia, carbon dimer and ethylene for various methods and basis sets. The TBEs have been extracted from Refs. 59 and 61 on the same geometries. See the supporting information for total energies.

\begin{tabular}{|c|c|c|c|c|c|c|c|c|c|c|c|c|c|c|c|}
\hline \multirow[b]{3}{*}{ Molecule } & \multirow[b]{3}{*}{ Transition } & \multirow[b]{3}{*}{ Nature } & \multirow[b]{3}{*}{ TBE } & \multicolumn{12}{|c|}{ Deviation with respect to TBE } \\
\hline & & & & \multicolumn{3}{|c|}{ exFCI } & \multicolumn{3}{|c|}{ exFCI+PBEot } & \multicolumn{3}{|c|}{ exFCI+PBE-UEG } & \multicolumn{3}{|c|}{ exFCI+LDA } \\
\hline & & & & $\overline{\text { AVDZ }}$ & AVTZ & $\overline{\text { AVQZ }}$ & AVDZ & AVTZ & $\overline{\text { AVQZ }}$ & AVDZ & AVTZ & $\overline{\text { AVQZ }}$ & AVDZ & AVTZ & $\overline{A V Q Z}$ \\
\hline \multirow[t]{6}{*}{ Water } & $1^{1} A_{1} \rightarrow 1^{1} B_{1}$ & Ryd. & $7.70^{\mathrm{a}}$ & -0.17 & -0.07 & -0.02 & 0.01 & 0.00 & 0.02 & -0.02 & -0.01 & 0.00 & -0.04 & -0.01 & $\tilde{0.01}$ \\
\hline & $1^{1} A_{1} \rightarrow 1^{1} A_{2}$ & Ryd. & $9.47^{\mathrm{a}}$ & -0.15 & -0.06 & -0.01 & 0.03 & 0.01 & 0.03 & 0.00 & 0.00 & 0.02 & -0.03 & 0.00 & 0.00 \\
\hline & $1^{1} A_{1} \rightarrow 2^{1} A_{1}$ & Ryd. & $9.97^{\mathrm{a}}$ & -0.03 & 0.02 & 0.06 & 0.13 & 0.08 & 0.09 & 0.10 & 0.07 & 0.08 & 0.09 & 0.07 & 0.03 \\
\hline & $1^{1} A_{1} \rightarrow 1^{3} B_{1}$ & Ryd. & $7.33^{\mathrm{a}}$ & -0.19 & -0.08 & -0.03 & 0.02 & 0.00 & 0.02 & 0.05 & 0.01 & 0.02 & 0.00 & 0.00 & 0.04 \\
\hline & $1^{1} A_{1} \rightarrow 1^{3} A_{2}$ & Ryd. & $9.30^{\mathrm{a}}$ & -0.16 & -0.06 & -0.01 & 0.04 & 0.02 & 0.04 & 0.07 & 0.03 & 0.04 & 0.03 & 0.03 & 0.04 \\
\hline & $1^{1} A_{1} \rightarrow 1^{3} A_{1}$ & Ryd. & $9.59^{\mathrm{a}}$ & -0.11 & -0.05 & -0.01 & 0.07 & 0.02 & 0.03 & 0.09 & 0.03 & 0.03 & 0.06 & 0.03 & 0.04 \\
\hline \multirow[t]{5}{*}{ Ammonia } & $1^{1} A_{1} \rightarrow 1^{1} A_{2}$ & Ryd. & $6.66^{\mathrm{a}}$ & -0.18 & -0.07 & -0.04 & -0.04 & -0.02 & -0.01 & -0.07 & -0.03 & -0.02 & -0.07 & -0.03 & -0.02 \\
\hline & $1^{1} A_{1} \rightarrow 1^{1} E$ & Ryd. & $8.21^{\mathrm{a}}$ & -0.13 & -0.05 & -0.02 & 0.01 & 0.00 & 0.01 & -0.03 & -0.01 & 0.00 & -0.03 & 0.00 & 0.00 \\
\hline & $1^{1} A_{1} \rightarrow 2^{1} A_{1}$ & Ryd. & $8.65^{\mathrm{a}}$ & 1.03 & 0.68 & 0.47 & 1.17 & 0.73 & 0.50 & 1.12 & 0.72 & 0.49 & 1.11 & 0.71 & 0.49 \\
\hline & $1^{1} A_{1} \rightarrow 2^{1} A_{2}$ & Ryd. & $8.65^{\mathrm{b}}$ & 1.22 & 0.77 & 0.59 & 1.36 & 0.83 & 0.62 & 1.33 & 0.81 & 0.61 & 1.32 & 0.81 & 0.61 \\
\hline & $1^{1} A_{1} \rightarrow 1^{3} A_{2}$ & Ryd. & $9.19^{\mathrm{a}}$ & -0.18 & -0.06 & -0.03 & -0.03 & 0.00 & -0.02 & -0.07 & -0.02 & -0.03 & -0.07 & -0.01 & -0.03 \\
\hline \multirow[t]{2}{*}{ Carbon dimer } & $1^{1} \Sigma_{g}^{+} \rightarrow 1^{1} \Delta_{g}$ & Val. & $2.04^{\mathrm{c}}$ & 0.17 & 0.05 & 0.02 & 0.04 & 0.00 & 0.00 & 0.15 & 0.04 & 0.02 & 0.17 & 0.05 & 0.02 \\
\hline & $1^{1} \Sigma_{g}^{+} \rightarrow 2^{1} \Sigma_{g}^{+}$ & Val. & $2.38^{\mathrm{c}}$ & 0.12 & 0.04 & 0.02 & 0.00 & 0.00 & 0.00 & 0.11 & 0.03 & 0.02 & 0.13 & 0.04 & 0.02 \\
\hline \multirow[t]{6}{*}{ Ethylene } & $1^{1} A_{1 g} \rightarrow 1^{1} B_{3 u}$ & Ryd. & $7.43^{\mathrm{c}}$ & -0.12 & -0.04 & & -0.05 & -0.01 & & -0.04 & -0.01 & & -0.02 & 0.00 & \\
\hline & $1^{1} A_{1 g} \rightarrow 1{ }^{1} B_{1 u}$ & Val. & $7.92^{\mathrm{c}}$ & 0.01 & 0.01 & & 0.00 & 0.00 & & 0.06 & 0.03 & & 0.06 & 0.03 & \\
\hline & $1^{1} A_{1 g} \rightarrow 1^{1} B_{1 g}$ & Ryd. & $8.10^{c}$ & -0.1 & -0.02 & & -0.03 & 0.00 & & -0.02 & 0.00 & & 0.00 & 0.01 & \\
\hline & $1^{1} A_{1 g} \rightarrow 1^{3} B_{1 u}$ & Val. & $4.54^{\mathrm{c}}$ & 0.01 & 0.00 & & 0.05 & 0.03 & & 0.08 & 0.04 & & 0.07 & 0.04 & \\
\hline & $1^{1} A_{1 g} \rightarrow 1^{3} B_{3 u}$ & Val. & $7.28^{\mathrm{d}}$ & -0.12 & -0.04 & & -0.04 & 0.00 & & 0.00 & 0.00 & & 0.00 & 0.02 & \\
\hline & $1^{1} A_{1 g} \rightarrow 1^{3} B_{1 g}$ & Val. & $8.00^{\mathrm{d}}$ & -0.07 & -0.01 & & 0.00 & 0.03 & & 0.04 & 0.03 & & 0.05 & 0.04 & \\
\hline
\end{tabular}

${ }^{\mathrm{a}}$ exFCI/AVQZ data corrected with the difference between $\mathrm{CC} 3 / \mathrm{d}-\mathrm{AV} 5 \mathrm{Z}$ and exFCI/AVQZ values. ${ }^{59} \mathrm{~d}-\mathrm{AV} 5 \mathrm{Z}$ is the doubly augmented V5Z basis set.

${ }^{\mathrm{b}}$ exFCI/AVTZ data corrected with the difference between CC3/d-AV5Z and exFCI/AVTZ values. ${ }^{59}$

${ }^{c}$ exFCI/CBS obtained from the exFCI/AVTZ and exFCI/AVQZ data of Ref. 61.

$\mathrm{d}$ exFCI/AVDZ data corrected with the difference between CC3/d-AV5Z and exFCI/AVDZ values. ${ }^{59}$
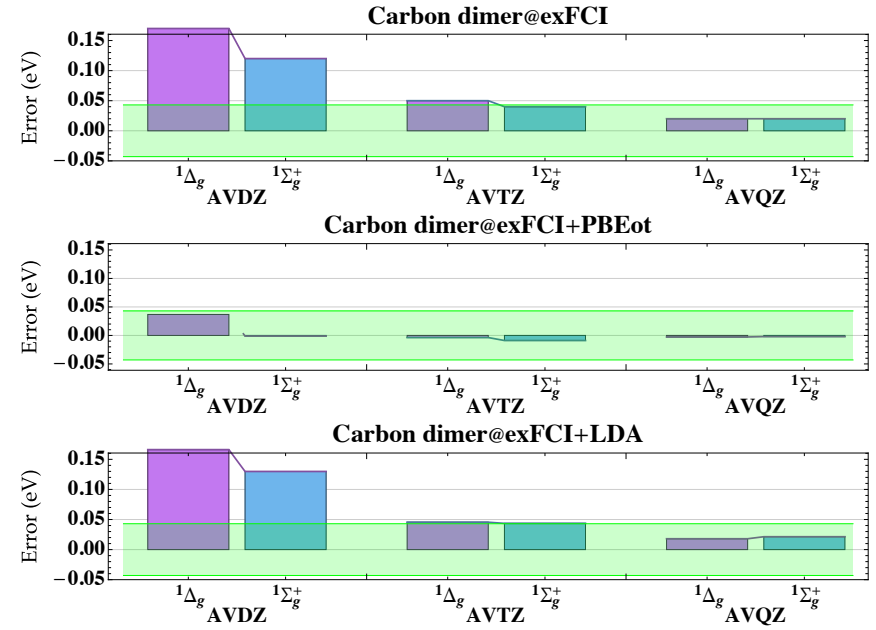

FIG. 4. Error in vertical excitation energies (in eV) for two doublyexcited states of the carbon dimer for various basis sets and methods. The green region corresponds to chemical accuracy (i.e., error below $1 \mathrm{kcal} / \mathrm{mol}$ or $0.043 \mathrm{eV}$ ). See the supporting information for raw data.

Regarding now the differential effect of the basis-set correction in the special case of the two ${ }^{1} \Sigma_{g}^{+}$states studied here, we observe that:

- $\mu^{\mathcal{B}}(z)$ has the same overall behavior for the two states, with slightly more fine structure in the case of the ground state. Such feature is consistent with the fact that the two states considered are both of $\Sigma_{g}^{+}$symmetry and of valence character.
- $n_{2}(z)$ is overall larger in the excited state, specially in the bonding and outer regions. This is can be explained by the nature of the electronic transition which qualitatively corresponds to a double excitation from $\pi$ to $\sigma$ orbitals, therefore increasing the overall electronic population on the bond axis.

- The energetic correction clearly stabilizes preferentially the excited state rather than the ground state, illustrating that short-range correlation effects are more pronounced in the former than in the latter. This is linked to the larger values of the excited-state on-top pair density.

\section{Ethylene}

As a final example, we consider the ethylene molecule, yet another system which has been particularly scrutinized theoretically using high-level ab initio methods. ${ }^{72,88-98}$ We refer the interested reader to the work of Feller et al. ${ }^{98}$ for an exhaustive investigation dedicated to the excited states of ethylene using state-of-the-art CI calculations. In the present context, ethylene is a particularly interesting system as it contains a mixture of valence and Rydberg excited states. Our basis-set corrected vertical excitation energies are gathered in Table II and depicted in Fig. 6. The exFCI+PBEot/AVDZ excitation energies are at near chemical accuracy and the errors drop further when one goes to the triple- $\zeta$ basis. Consistently with the previous examples, the LDA and PBE-UEG functionals are 



FIG. 5. $\mu^{\mathcal{B}}$ (left), $n^{\mathcal{B}} \bar{\varepsilon}_{\mathrm{c}, \text { md }}^{\text {sr,PBEot }}$ (center) and $n_{2}^{\mathcal{B}}$ (right) along the molecular axis ( $z$ ) for the ground state (black curve) and second doubly-excited state (red curve) of $\mathrm{C}_{2}$ for various basis sets $\mathcal{B}$. The two electronic states are both of $\Sigma_{g}^{+}$symmetry. The carbon nuclei are located at $z= \pm 1.180 \mathrm{bohr}$ and are represented by the thin black lines.

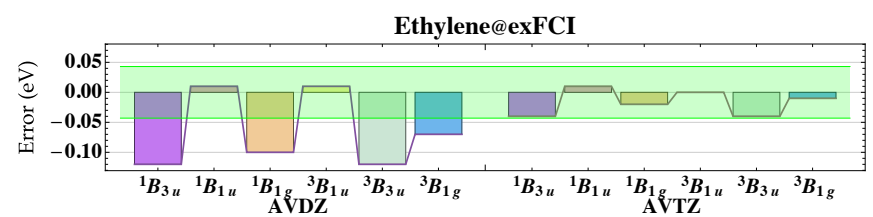

Ethylene@exFCI+PBEot



Ethylene@exFCI+LDA

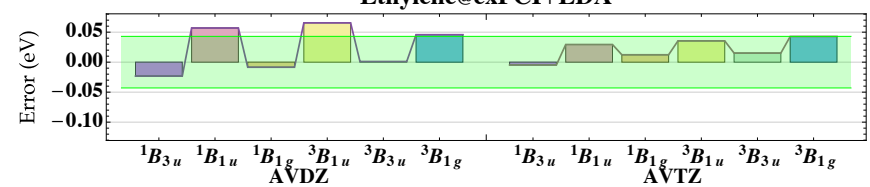

FIG. 6. Error in vertical excitation energies (in eV) of ethylene for various basis sets and methods. The green region corresponds to chemical accuracy (i.e., error below $1 \mathrm{kcal} / \mathrm{mol}$ or $0.043 \mathrm{eV}$ ). See the supporting information for raw data.

slightly less accurate, although they still correct the excitation energies in the right direction.

\section{CONCLUSION}

We have shown that, by employing the recently proposed density-based basis-set correction developed by some of the authors, ${ }^{19}$ one can obtain, using sCI methods, chemically accurate excitation energies with typically augmented double- $\zeta$ basis sets. This nicely complements our recent investigation on ground-state properties, ${ }^{20}$ which has evidenced that one recovers quintuple- $\zeta$ quality atomization and correlation energies with triple- $\zeta$ basis sets. The present study clearly shows that, for very diffuse excited states, the present correction relying on short-range correlation functionals from RS-DFT might not be enough to catch the radial incompleteness of the one-electron basis set. Also, in the case of multireference systems, we have evidenced that the PBEot functional, which uses an accurate on-top pair density, is more appropriate than the LDA and PBE-UEG functionals relying on the UEG on-top pair density. We are currently investigating the performance of the present basis-set correction for strongly correlated systems and we hope to report on this in the near future.

\section{SUPPORTING INFORMATION AVAILABLE}

See supporting information for geometries and additional information (including total energies and energetic correction of the various functionals).

\section{ACKNOWLEDGMENTS}

PFL would like to thank Denis Jacquemin for numerous discussions on excited states. This work was performed using HPC resources from GENCI-TGCC (Grant No. 2018A0040801738), CALMIP (Toulouse) under allocation 201918005 and the Jarvis-Alpha cluster from the Institut Parisien de Chimie Physique et Théorique.

${ }^{1}$ Kutzelnigg, W. R12-Dependent Terms In The Wave Function As Closed Sums Of Partial Wave Amplitudes For Large L. Theor. Chim. Acta 1985, 68, 445.

${ }^{2}$ Kutzelnigg, W.; Klopper, W. Wave Functions With Terms Linear In The Interelectronic Coordinates To Take Care Of The Correlation Cusp. I. General Theory. F. Chem. Phys. 1991, 94, 1985.

${ }^{3}$ Noga, J.; Kutzelnigg, W. Coupled Cluster Theory That Takes Care Of The Correlation Cusp By Inclusion Of Linear Terms In The Interelectronic Coordinates. F. Chem. Phys. 1994, 101, 7738.

${ }^{4}$ Ten-no, S. Explicitly correlated wave functions: summary and perspective. Theor. Chem. Acc. 2012, 131, 1070. 
${ }^{5}$ Ten-no, S.; Noga, J. Explicitly Correlated Electronic Structure Theory From R12/F12 Ansatze. WIREs Comput. Mol. Sci. 2012, 2, 114.

${ }^{6}$ Hattig, C.; Klopper, W.; Kohn, A.; Tew, D. P. Explicitly Correlated Electrons in Molecules. Chem. Rev. 2012, 112, 4

${ }^{7}$ Kong, L.; Bischo, F. A.; Valeev, E. F. Explicitly Correlated R12/F12 Methods for Electronic Structure. Chem. Rev. 2012, 112, 75.

${ }^{8}$ Grüneis, A.; Hirata, S.; Ohnishi, Y.-Y.; Ten-no, S. Perspective: Explicitly Correlated Electronic Structure Theory For Complex Systems. 7. Chem. Phys. 2017, 146, 080901.

${ }^{9}$ Ma, Q.; Werner, H.-J. Explicitly Correlated Local Coupled-Cluster Methods Using Pair Natural Orbitals. WIREs Comput. Mol. Sci. 2018, 8, e1371.

${ }^{10}$ Tew, D. P.; Klopper, W.; Neiss, C.; Hattig, C. Quintuple- $\zeta$ Quality CoupledCluster Correlation Energies With Triple- $\zeta$ Basis Sets. Phys. Chem. Chem. Phys. 2007, 9, 1921.

${ }^{11}$ Fliegl, H.; Hattig, C.; Klopper, W. Coupled-cluster response theory with linear-r12 corrections: The CC2-R12 model for excitation energies. f. Chem. Phys. 2006, 124, 044112.

${ }^{12}$ Neiss, C.; Hattig, C.; Klopper, W. Extensions of r12 corrections to CC2-R12 for excited states. F. Chem. Phys. 2006, 125, 064111.

${ }^{13}$ Hanauer, M.; Kohn, A. Response properties with explicitly correlated coupled-cluster methods using a Slater-type correlation factor and cusp conditions. F. Chem. Phys. 2009, 131, 124118.

${ }^{14} \mathrm{Kohn}, \mathrm{A}$. A modified ansatz for explicitly correlated coupled-cluster wave functions that is suitable for response theory. F. Chem. Phys. 2009, 130, 104104.

${ }^{15}$ Shiozaki, T.; Werner, H.-J. Communication: Second-order multireference perturbation theory with explicit correlation: CASPT2-F12. f. Chem. Phys. 2010, 133, 141103.

${ }^{16}$ Shiozaki, T.; Knizia, G.; Werner, H.-J. Explicitly correlated multireference configuration interaction: MRCI-F12. f. Chem. Phys. 2011, 134, 034113.

${ }^{17}$ Shiozaki, T.; Werner, H.-J. Explicitly correlated multireference configuration interaction with multiple reference functions: Avoided crossings and conical intersections. f. Chem. Phys. 2011, 134, 184104.

${ }^{18}$ Shiozaki, T.; Werner, H.-J. Multireference explicitly correlated F12 theories. Mol. Phys. 2013, 111, 607-630.

${ }^{19}$ Giner, E.; Pradines, B.; Ferté, A.; Assaraf, R.; Savin, A.; Toulouse, J. Curing Basis-Set Convergence Of Wave-Function Theory Using Density-Functional Theory: A Systematically Improvable Approach. f. Chem. Phys. 2018, 149, 194301.

${ }^{20}$ Loos, P. F.; Pradines, B.; Scemama, A.; Toulouse, J.; Giner, E. A DensityBased Basis-Set Correction for Wave Function Theory. 7. Phys. Chem. Lett. 2019, 10, 2931-2937.

${ }^{21}$ Savin, A. On Degeneracy, Near Degeneracy and Density Functional Theory. In Recent Developments of Modern Density Functional Theory; Seminario, J. M., Ed.; Elsevier: Amsterdam, 1996; pp 327-357.

${ }^{22}$ Leininger, T.; Stoll, H.; Werner, H.-J.; Savin, A. Combining Long-Range Configuration Interaction With Short-Range Density Functionals. Chem. Phys. Lett. 1997, 275, 151.

${ }^{23}$ Toulouse, J.; Colonna, F.; Savin, A. Long-Range-Short-Range Separation Of The Electron-Electron Interaction In Density-Functional Theory. Phys. Rev. A 2004, 70, 062505.

${ }^{24}$ Toulouse, J.; Savin, A.; Flad, H.-J. Short-range exchange-correlation energy of a uniform electron gas with modified electron-electron interaction. Int. 7. Quantum Chem. 2004, 100, 1047.

${ }^{25}$ Ángyán, J. G.; Gerber, I. C.; Savin, A.; Toulouse, J. van der Waals Forces In Density Functional Theory: Perturbational Long-Range Electron Interaction Corrections. Phys. Rev. A 2005, 72, 012510.

${ }^{26}$ Goll, E.; Werner, H.-J.; Stoll, H. A Short-Range Gradient-Corrected Density Functional In Long-Range Coupled-Cluster Calculations For Rare Gas Dimers. Phys. Chem. Chem. Phys. 2005, 7, 3917.

${ }^{27}$ Paziani, S.; Moroni, S.; Gori-Giorgi, P.; Bachelet, G. B. Local-Spin-Density Functional For Multideterminant Density Functional Theory. Phys. Rev. $B$ 2006, 73, 155111.

${ }^{28}$ Fromager, E.; Toulouse, J.; Jensen, H. J. A. On The Universality Of The Long-/Short-Range Separation In Multiconfigurational Density-Functional Theory. F. Chem. Phys. 2007, 126, 074111.

${ }^{29}$ Toulouse, J.; Gerber, I. C.; Jansen, G.; Savin, A.; Ángyán, J. G. AdiabaticConnection Fluctuation-Dissipation Density-Functional Theory Based On Range Separation. Phys. Rev. Lett. 2009, 102, 096404.
${ }^{30}$ Janesko, B. G.; Henderson, T. M.; Scuseria, G. E. Long-Range-Corrected Hybrids Including Random Phase Approximation Correlation. f. Chem. Phys. 2009, 130, 081105.

${ }^{31}$ Fromager, E.; Cimiraglia, R.; Jensen, H. J. A. Merging Multireference Perturbation And Density-Functional Theories By Means Of Range Separation Potential Curves For $\mathrm{Be}_{2}, \mathrm{Mg}_{2}$, And Ca 2 . Phys. Rev. A 2010, 81, 024502.

${ }^{32}$ Toulouse, J.; Zhu, W.; Savin, A.; Jansen, G.; Ángyán, J. G. Closed-Shell Ring Coupled Cluster Doubles Theory With Range Separation Applied On Weak Intermolecular Interactions. f. Chem. Phys. 2011, 135, 084119.

${ }^{33}$ Mussard, B.; Reinhardt, P.; Ángyán, J. G.; Toulouse, J. Spin-Unrestricted Random-Phase Approximation With Range Separation: Benchmark On Atomization Energies And Reaction Barrier Heights. f. Chem. Phys. 2015, 142, 154123, Erratum: J. Chem. Phys. 142, 219901 (2015).

${ }^{34}$ Hedegård, E. D.; Knecht, S.; Kielberg, J. S.; Jensen, H. J. A.; Reiher, M. Density Matrix Renormalization Group With Efficient Dynamical Electron Correlation Through Range Separation. f. Chem. Phys. 2015, 142, 224108.

${ }^{35}$ Hedegård, E. D.; Toulouse, J.; Jensen, H. J. A. Multiconfigurational ShortRange Density-Functional Theory For Open-Shell Systems. 7. Chem. Phys. 2018, 148, 214103.

${ }^{36}$ Ferté, A.; Giner, E.; Toulouse, J. Range-Separated Multideterminant DensityFunctional Theory With A Short-Range Correlation Functional Of The On-Top Pair Density. f. Chem. Phys. 2019, 150, 084103.

${ }^{37}$ Parr, R. G.; Yang, W. Density-Functional Theory of Atoms and Molecules; Oxford University Press: New York, 1989.

${ }^{38}$ Szabo, A.; Ostlund, N. S. Modern Quantum Chemistry: Introduction to Advanced Electronic Structure Theory; Dover: New York, 1996.

${ }^{39} \mathrm{Kato}$, T. On the eigenfunctions of many-particle systems in quantum mechanics. Comm. Pure Appl. Math. 1957, 10, 151.

${ }^{40}$ Franck, O.; Mussard, B.; Luppi, E.; Toulouse, J. Basis Convergence Of RangeSeparated Density-Functional Theory. f. Chem. Phys. 2015, 142, 074107.

${ }^{41}$ Morgan III, J. D.; Kutzelnigg, W. Hund's rules, the alternating rule, and symmetry holes. f. Phys. Chem. 1993, 97, 2425.

${ }^{42}$ Kutzelnigg, W.; Morgan III, J. D. Hund's rules. Z. Phys. D 1996, 36, 197.

${ }^{43} \mathrm{Tew}$, D. P. Second-order coalescence conditions of molecular wave functions. 7. Chem. Phys. 2008, 129, 014104.

${ }^{44}$ Loos, P. F.; Gill, P. M. W. Excited states of spherium. Mol. Phys. 2010, 108, 2527.

${ }^{45}$ Loos, P. F.; Bloomfield, N. J.; Gill, P. M. W. F. Chem. Phys. 2015, 143, 181101

${ }^{46}$ Toulouse, J.; Gori-Giorgi, P.; Savin, A. A Short-Range Correlation Energy Density Functional With Multi-Determinantal Reference. Theor. Chem. Acc. 2005, 114, 305.

${ }^{47}$ Hohenberg, P.; Kohn, W. Inhomogeneous Electron Gas. Phys. Rev. 1964, 136, B 864

${ }^{48}$ Kohn, W.; Sham, L. J. Self-Consistent Equations Including Exchange and Correlation Effects. Phys. Rev. 1965, 140, A1133.

${ }^{49}$ Loos, P.-F.; Gill, P. M. W. The Uniform Electron Gas. WIREs Comput. Mol. Sci. 2016, 6, 410 .

${ }^{50}$ Perdew, J. P.; Burke, K.; Ernzerhof, M. Generalized Gradient Approximation Made Simple. Phys. Rev. Lett. 1996, 77, 3865.

${ }^{51}$ Gori-Giorgi, P.; Savin, A. Properties Of Short-Range And Long-Range Correlation Energy Density Functionals From Electron-Electron Coalescence. Phys. Rev. A 2006, 73, 032506.

${ }^{52}$ Huron, B.; Malrieu, J.; Rancurel, P. Iterative Perturbation Calculations Of Ground And Excited State Energies From Multiconfigura- Tional ZerothOrder Wavefunctions. 7. Chem. Phys. 1973, 58, 5745.

${ }^{53}$ Giner, E.; Scemama, A.; Caffarel, M. Using Perturbatively Selected Configuration Interaction In Quantum Monte Carlo Calculations. Can. F. Chem. 2013, 91, 879.

${ }^{54}$ Giner, E.; Scemama, A.; Caffarel, M. Fixed-Node Diffusion Monte Carlo Potential Energy Curve Of The Fluorine Molecule F2 Using Selected Configuration Interaction Trial Wavefunctions. F. Chem. Phys. 2015, 142.

${ }^{55}$ Scemama, A.; Caffarel, M.; Benali, A.; Jacquemin, D.; Loos, P. F. Influence of Pseudopotentials on Excitation Energies From Selected Configuration Interaction and Diffusion Monte Carlo. Res. Chem. 2019, in press.

${ }^{56}$ Holmes, A. A.; Umrigar, C. J.; Sharma, S. Excited States Using Semistochastic Heat-Bath Configuration Interaction. f. Chem. Phys. 2017, 147, 164111.

${ }^{57}$ Garniron, Y.; Gasperich, K.; Applencourt, T.; Benali, A.; Ferté, A.; Paquier, J.; Pradines, B.; Assaraf, R.; Reinhardt, P.; Toulouse, J.; Barbaresco, P.; Renon, N.; David, G.; Malrieu, J. P.; Véril, M.; Caffarel, M.; Loos, P. F.; Giner, E.; Scemama, A. Quantum Package 2.0: A Open-Source Determinant- 
Driven Suite Of Programs. 7. Chem. Theory Comput. 2019, 15, 3591.

${ }^{58}$ Scemama, A.; Garniron, Y.; Caffarel, M.; Loos, P. F. Deterministic Construction Of Nodal Surfaces Within Quantum Monte Carlo: The Case Of Fes. $\mathcal{F}$. Chem. Theory Comput. 2018, 14, 1395.

${ }^{59}$ Loos, P.-F.; Scemama, A.; Blondel, A.; Garniron, Y.; Caffarel, M.; Jacquemin, D. A Mountaineering Strategy to Excited States: Highly Accurate Reference Energies and Benchmarks. f. Chem. Theory Comput. 2018, $14,4360-4379$.

${ }^{60}$ Scemama, A.; Benali, A.; Jacquemin, D.; Caffarel, M.; Loos, P.-F. Excitation Energies From Diffusion Monte Carlo Using Selected Configuration Interaction Nodes. f. Chem. Phys. 2018, 149, 034108.

${ }^{61}$ Loos, P.-F.; Boggio-Pasqua, M.; Scemama, A.; Caffarel, M.; Jacquemin, D. Reference Energies for Double Excitations. 7. Chem. Theory Comput. 2019, $15,1939-1956$.

${ }^{62}$ Dasgupta, S.; Herbert, J. M. Standard grids for high-precision integration of modern density functionals: SG-2 and SG-3. f. Comput. Chem. 2017, 38, 869-882.

${ }^{63}$ Sherrill, C. D.; Leininger, M. L.; Huis, T. J. V.; Schaefer, H. F. Structures and vibrational frequencies in the full configuration interaction limit: Predictions for four electronic states of methylene using a triple-zeta plus double polarization (TZ2P) basis. F. Chem. Phys. 1998, 108, 1040.

${ }^{64} \mathrm{H}$. F. Schaeffer III, Methylene: A paradigm for computational quantum chemistry. 1986, 1100-1107.

${ }^{65}$ Bauschlicher, C. W.; Taylor, P. R. A full CI treatment of the 1A1-3B1 separation in methylene. 7. Chem. Phys. 1986, 85, 6510-6512.

${ }^{66}$ Jensen, P.; Bunker, P. R. The potential surface and stretching frequencies of X3B1 methylene $(\mathrm{CH} 2)$ determined from experiment using the Morse oscillator-rigid bender internal dynamics Hamiltonian. f. Chem. Phys. 1988, 89, 1327.

${ }^{67}$ Sherrill, C. D.; Van Huis, T. J.; Yamaguchi, Y.; Schaefer, H. F. Full configuration interaction benchmarks for the states of methylene. F. Mol. Struct. (THEOCHEM) 1997, 400, 139-156.

${ }^{68}$ Abrams, M. L.; Sherrill, C. D. Full Configuration Interaction Potential Energy Curves for the $\mathrm{X} 1 \Sigma_{g}^{+}$, B $1 \Delta_{g}$, and $\mathrm{B}^{\prime} 1 \Sigma_{g}^{+}$States of $\mathrm{C}_{2}$ : A Challenge for Approximate Methods. F. Chem. Phys. 2004, 121, 9211-9219.

${ }^{69}$ Abrams, M. L.; Sherrill, C. D. Important configurations in configuration interaction and coupled-cluster wave functions. Chem. Phys. Lett. 2005, 412, $121-124$

${ }^{70}$ Zimmerman, P. M.; Toulouse, J.; Zhang, Z.; Musgrave, C. B.; Umrigar, C. J. Excited states of methylene from quantum Monte Carlo. F. Chem. Phys. 2009, 131, 124103.

${ }^{71}$ Gour, J. R.; Piecuch, P.; Wloch, M. Comparison of the completely renormalized equation-of-motion coupled-cluster and Quantum Monte Carlo results for the low-lying electronic states of methylene. Mol. Phys. 2010, 108, 2633-2646.

${ }^{72}$ Chien, A. D.; Holmes, A. A.; Otten, M.; Umrigar, C. J.; Sharma, S.; Zimmerman, P. M. Excited States of Methylene, Polyenes, and Ozone from Heat-Bath Configuration Interaction. f. Phys. Chem. A 2018, 122, 27142722 .

${ }^{73}$ Helgaker, T.; Jørgensen, P.; Olsen, J. Molecular Electronic-Structure Theory; Wiley: Chichester, 2002.

${ }^{74}$ Loos, P.-F.; Galland, N.; Jacquemin, D. Theoretical 0-0 Energies with Chemical Accuracy. F. Phys. Chem. Lett. 2018, 9, 4646-4651.

${ }^{75}$ Loos, P. F.; Jacquemin, D. Chemically accurate $0-0$ energies with not-soaccurate excited state geometries. f. Chem. Theory Comput. 2019, 15, 24812491.

${ }^{76}$ Cai, Z.-L.; Tozer, D. J.; Reimers, J. R. Time-Dependent Density-Functional Determination of Arbitrary Singlet and Triplet Excited-State Potential Energy Surfaces: Application to the Water Molecule. f. Chem. Phys. 2000, 113, 7084-7096.
${ }^{77}$ Rubio, M.; Serrano-Andrés, L.; Merchán, M. Excited States of the Water Molecule: Analysis of the Valence and Rydberg Character. F. Chem. Phys. 2008, 128, 104305 .

${ }^{78} \mathrm{Li}$, X.; Paldus, J. Multi-Reference State-Universal Coupled-Cluster Approaches to Electronically Excited States. 7. Chem. Phys. 2011, 134, 214118.

${ }^{79}$ Schwabe, T.; Goerigk, L. Time-Dependent Double-Hybrid Density Functionals with Spin-Component and Spin-Opposite Scaling. 7. Chem. Theory Comput. 2017, 13, 4307-4323.

${ }^{80}$ Bartlett, R. J.; Bene, J. E. D.; Perera, S.; Mattie, R.-P. Ammonia: The Prototypical Lone Pair Molecule. f. Mol. Struct. (THEOCHEM) 1997, 400, 157-168.

${ }^{81}$ Varandas, A. J. C. Extrapolation to the Complete-Basis-Set Limit and the Implications of Avoided Crossings: The X $\Sigma 1 \mathrm{~g}+, \mathrm{B} \Delta 1 \mathrm{~g}$, and $\mathrm{B}^{\prime} \Sigma 1 \mathrm{~g}+$ States of C2. F. Chem. Phys. 2008, 129, 234103.

${ }^{82}$ Purwanto, W.; Zhang, S.; Krakauer, H. Excited State Calculations Using Phaseless Auxiliary-Field Quantum Monte Carlo: Potential Energy Curves of Low-Lying C2 Singlet States. F. Chem. Phys. 2009, 130, 094107.

${ }^{83}$ Angeli, C.; Cimiraglia, R.; Pastore, M. A Comparison of Various Approaches in Internally Contracted Multireference Configuration Interaction: The Carbon Dimer as a Test Case. Mol. Phys. 2012, 110, 2963-2968.

${ }^{84}$ Booth, G. H.; Cleland, D.; Thom, A. J. W.; Alavi, A. Breaking The Carbon Dimer: The Challenges Of Multiple Bond Dissociation With Full Configuration Interaction Quantum Monte Carlo Methods. F. Chem. Phys. 2011, 135, 084104.

${ }^{85}$ Sharma, S. A General Non-Abelian Density Matrix Renormalization Group Algorithm with Application to the $\mathrm{C}_{2}$ Dimer. f. Chem. Phys. 2015, 142, 024107.

${ }^{86}$ Sokolov, A. Y.; Chan, G. K.-L. A Time-Dependent Formulation of MultiReference Perturbation Theory. f. Chem. Phys. 2016, 144, 064102.

${ }^{87}$ Varandas, A. J. C.; Rocha, C. M. R. $C_{n}(n=2-4)$ : Current Status. Philos. Trans. R. Soc. Math. Phys. Eng. Sci. 2018, 376, 20170145.

${ }^{88}$ Serrano-Andrés, L.; Merchán, M.; Nebot-Gil, I.; Lindh, R.; Roos, B. O. Towards an Accurate Molecular Orbital Theory for Excited States: Ethene, Butadiene, and Hexatriene. 7. Chem. Phys. 1993, 98, 3151-3162.

${ }^{89}$ Watts, J. D.; Gwaltney, S. R.; Bartlett, R. J. Coupled-cluster Calculations of the Excitation Energies of Ethylene, Butadiene, and Cyclopentadiene. 7 . Chem. Phys. 1996, 105, 6979-6988.

${ }^{90}$ Wiberg, K. B.; de Oliveria, A. E.; Trucks, G. A Comparison of the Electronic Transition Energies for Ethene, Isobutene, Formaldehyde, and Acetone Calculated Using RPA, TDDFT, and EOM-CCSD. Effect of Basis Sets. F. Phys. Chem. A 2002, 106, 4192-4199.

${ }^{91}$ Barbatti, M.; Paier, J.; Lischka, H. Photochemistry Of Ethylene: A Multireference Configuration Interaction Investigation Of The Excited-State Energy Surfaces. F. Chem. Phys. 20004, 121, 11614.

${ }^{92}$ Angeli, C. On the Nature of the $\pi \rightarrow \pi^{*}$ Ionic Excited States: The V State of Ethene as a Prototype. f. Comput. Chem. 2008, 30, 1319-1333.

${ }^{93}$ Schreiber, M.; Silva-Junior, M. R.; Sauer, S. P. A.; Thiel, W. Benchmarks for Electronically Excited States: CASPT2, CC2, CCSD and CC3. 7. Chem. Phys. 2008, 128, 134110 .

${ }^{94}$ Silva-Junior, M. R.; Schreiber, M.; Sauer, S. P. A.; Thiel, W. Benchmarks of Electronically Excited States: Basis Set Effecs Benchmarks of Electronically Excited States: Basis Set Effects on CASPT2 Results. 7. Chem. Phys. 2010, 133, 174318

${ }^{95}$ Silva-Junior, M. R.; Sauer, S. P. A.; Schreiber, M.; Thiel, W. Basis Set Effects on Coupled Cluster Benchmarks of Electronically Excited States: CC3, CCSDR(3) and CC2. Mol. Phys. 2010, 108, 453-465.

${ }^{96}$ Angeli, C. An Analysis of the Dynamic $\sigma$ Polarization in the V State of Ethene. Int. F. Quantum Chem. 2010, 2436-2447.

${ }^{97}$ Daday, C.; Smart, S.; Booth, G. H.; Alavi, A.; Filippi, C. Full Configuration Interaction Excitations of Ethene and Butadiene: Resolution of an Ancient Question. F. Chem. Theory. Comput. 2012, 8, 4441-4451.

${ }^{98}$ Feller, D.; Peterson, K. A.; Davidson, E. R. A Systematic Approach to Vertically Excited States of Ethylene Using Configuration Interaction and Coupled Cluster Techniques. F. Chem. Phys. 2014, 141, 104302. 